\title{
RÉALISATION EXPÉRIMENTALE DU CYCLE COMPLET
}

\section{DU SCHISTOSOMA HAEMATOBIUM}

\author{
Par E. BRUMPT
}

Dans une communication faite à l'Académie de Médecine le 31 janvier dernier, en collaboration avec $\mathrm{S}$. Werblunsky, j'ai signalé la possibilité d'infecter expérimentalement des Bullinus contortus, originaires de Corse, avec l'urine de deux malades égyptiens souffrant de bilharziose vésicale. Depuis cette époque j'ai effectué de nombreuses expériences en partant de ces mollusques, dans le but de donner la bilharziose à divers animaux de laboratoire. J'ai réussi à infecter un Macacus cynomolgus (sujet unique), un rat blanc sur huit, neuf souris blanches sur dix et six hérissons jeunes ou adultes sur six. Par contre, je n'ai enregistré que des échecs en utilisant cinq jeunes chats, de 400 à 600 grammes, sept lapins de quelques mois et un jeune cobaye.

Les souris ont présenté soit des infections trop intenses, les faisant succomber avec de nombreux schistosomes jeunes dans le foie, soit des infections relativement légères passant à l'état chronique. Dans ce dernier cas, les animaux sacrifiés entre le $96^{\circ}$ et le $107^{\circ}$ jour après le début de l'expérience montraient à l'autopsie un foie cirrhotique très altéré, de couleur foncće, à surface irrégulière, avec de nombreux œufs et quelques vers adultes ainsi qu'une grande quantité de pigment noir (hémozoïne). Certaines souris avaient la rate hypertrophiée (1) avec des oufs nombreux dans un cas, plus rares dans d'autres.

(1) La rate est hypertrophiée chez les gens atteints de bilharziose sino-japonaise provoquée par le Schistosoma japonicum; elle semble habituellement hypertrophiée chez de nombreux sujets porteurs de Schistosoma mansoni aussi bien en Afrique qu'en Amérique. Un certain nombre d'auteurs attribuent même la splénomégalie égyptienne à une infection par le Schistosoma mansoni qui chez le même sujet provoque également une cirrhose du foie. Mes expériences, en montrant l'existence d'hypertrophie de la rate et la présence de nombreux oufs dans cet organe, même dans le cas des infections à Schistosoma hamatobium, consolident l'hypothèse de l'étiologie bilharzienne de la splénomégalie d'Egypte. La présence de foyers renfermant de nombreux œufs de Schistosoma hamatobium, tantôt tous normaux (fig. 3) tantôt tous anormaux, me permet de croire que, contrairement à l'opinion généralement admise, les œufs de bilharzies sont directement déposés dans les tissus des organes par les femelles susceptibles de sortir des petits vaisseaux sanguins pour pondre, et susceptibles d'y reitrer par effraction pour retrouver leur alimentation sanguine et leur habitat normal. Je ne crois pas que la distribution des œufs dans les organes puisse être expliquée par la migration des œufs pondus dans les veines ou entrainés par le courant sanguin après le départ des femelles qui bloquaient les vaisseaux.

Annales de Parasitologie, T. VI, No $4 .-1^{\text {er }}$ octobre 1928 , p. 440-446. 
Les poumons renfermaient parfois des vers adultes et des œufs. La vessie et l'intestin ne montraient pas d'œufs ou de vers dans les cas examinés.

Les hérissons présentent des infections intenses qu'ils supportent relativement bien. Trois hérissons ayant vécu plus de trois mois ont eu une dysenterie bilharzienne légère, caractérisée par la présence de glaires sanglantes et parfois même d'un véritable crachat rectal, riches en œufs à éperon polaire, à la surface de leurs déjections. Un de ces hérissons (681-IX) a été sacrifié 110 jours après le début de l'expérience. Les deux autres hérissons sont morts l'un (678-IX) le $118^{\circ}$ jour, l'autre (679IX) le $97^{\circ}$ jour, ce dernier à la suite d'un trop fort parasitisme expérimental par des nymphes de Dermacentor reticulatus. A l'autopsie de ces deux animaux, les seules lésions macroscopiques sont montrées par le foie dont la coloration brune est anormale, par la portion terminale de l'intestin (fig. 1) et par l'estomac dans un cas qui présentent un épaississement de la muqueuse et des hémorragies punctiformes ou linéaires, isolées ou confluentes. Les poumons, renfermant quelques strongles, présentent de légères lésions de bronchite vermineuse mais pas d'œufs de schistosome ni de vers adultes. Le foie et le pancréas sont infiltrés d'œufs et de pigment noir. La rate, non hypertrophiée, ne montre pas d'œufs à l'examen direct. La paroi du tube digestif présente des œufs en nombre variable depuis le début de l'œsophage jusqu'à environ -un centimètre de l'anus, mais

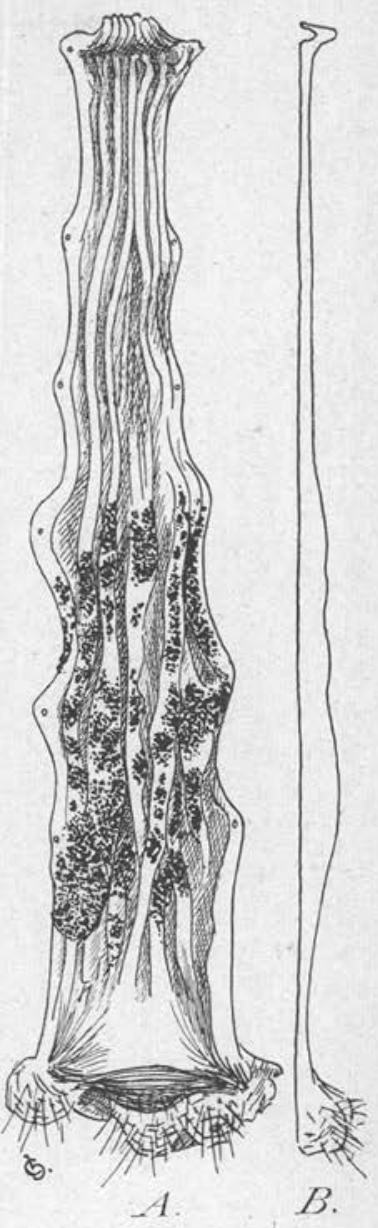

FIG. 1. - Intestin terminal du hérisson 681-1X. Grandeur naturelle. $A$, Intestin ouvert, montrant l'épaississement des parois et lıs hémorragies caractéristiques finissant en bas à deux centimètres de l'anus : $B$, le même, vu de profil pour montrer l'épaississcment au niveau des lésions. ils sont surtout très nombreux et décelés par des lésions macroscopiques dans l'intestin terminal (1) et l'estomac. Le raclage de la mu-

(1) Je signale en passant qu'il est impossible de déterminer macroscopiquement la limite du gros intestin et du grêle chez le hérisson. 
queuse buccale et de la langue n'a pas permis de déceler d'œufs. Des coupes histologiques dans le dernier quart de l'intestin terminal, au niveau des lésions signalées ci-dessus, permettent de trouver de nombreux îlots d'œufs et au-dessous, dans la musculeuse ou dans la séreuse, des schistosomes adultes mâles ou femelles. Le cerveau, les capsules surrénales et le rein, malgré la présence de cylindres dans

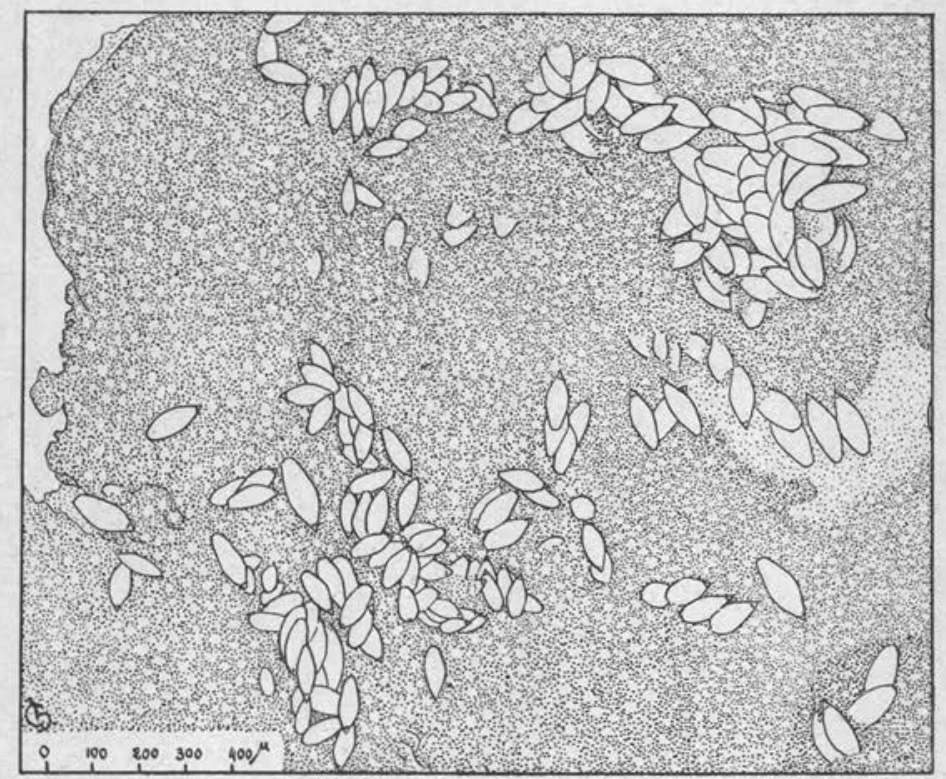

FIG. 2. - Fragment de pancréas du hérisson 678-IX, examiné à létat frais entre deux lames. Remarquer l'abondance des œufs dans certains champs microscopiques et les amas d'œufs constitués par des éléments d'aspect identique dans chaque îlot mais de structure et de dimensions variables dans des îlots différents. $\times 50$.

l'urine, ne présentaient pas d'œufs. Fait curieux, dans les deux cas la vessie était saine, aucun ouf n'a pu être mis en évidence par le raclage de la muqueuse. L'urine qui remplissait la vessie dans un cas était claire, sans œufs ; elle a été jetée accidentellement avant que la recherche du sucre ait pu être effectuée, recherche qui aurait été intéressante étant donnée l'intense infection du pancréas (fig. 2) de l'animal étudié (1).

(1) Dans son travail sur l'étiologie de la splénomégalie et la cirrhose hépatique égyptiennes, H.-B. Day (1924) admet que le diabète, fréquent en Egypte, peut être dû parfois aux bilharzies. Il signale le cas d'un sujet souffrant de bilharziose vési- 
Le dernier hérisson (681, IX), qui s'alimentait encore bien, a été sacrifiẻ le 12 juillet, 110 jours après le début de l'expérience. Les lésions macroscopiques du tube digestif étaient localisées à la partie terminale du gros intestin ainsi qu'au début de l'intestin grêle qui présentait des ecchymoses et de nombreux oufs, depuis le pylore jusqu'au vingt-cinquième centimètre au-dessous de lui. La vessie

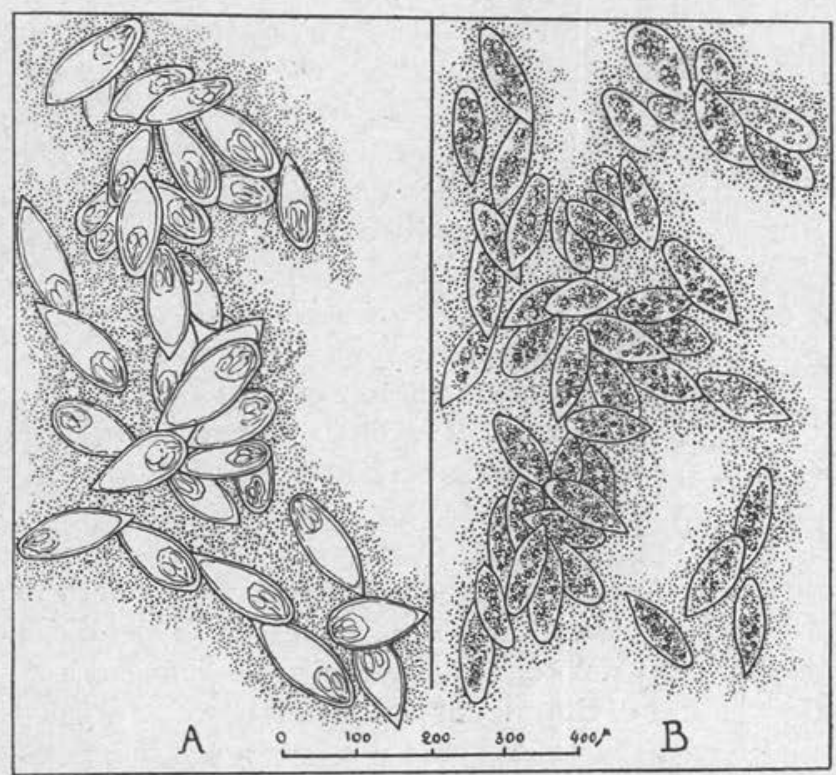

Fig. 3. - Même pancréas (hérisson 678-IX). A, îlot d'œufs normaux avec miracidium ; $B$, cufs de petite taille ne renfermant pas de miracidium. $\times 80$.

ne montrait ni lésions, ni œufs et l'urine, malgré l'existence de lésions pancréatiques et hépatiques intenses, ne renfermait ni sucre, ni albumine, ni corps acétoniques, bien que ce hérisson ait été soumis à un régime alimentaire mixte (soupe au pain et poumon de bœuf) depuis plusieurs mois.

Il est tout à fait intéressant de remarquer que les trois hérissons étudiés ci-dessus ont présenté des lésions intestinales très nettes, par lesquelles se faisait l'évacuation des œufs dans le milieu extérieur et aucune léșion vésicale, bien qu'ils aient été infectés en

cale, présentant au moment de l'examen 36 grammes de sucre par litre, guéri en quelques semaines de sa glycosurie, sans changement de régime alimentaire, par le traitement à l'émétique. Les lésions du pancréas provoquées par les cufs de biharzie sont peut-être responsables de ces cas. 
partant de mollusques contaminés par l'urine de deux malades égyptiens.

Je reviendrai dans un travail plus complet sur ces curieux tropismes du Schistosoma hæmatobium qui chez l'homme aussi, d'après les observations d'Ouzilleau, de Clapier, de Chestermann, de Nessmann et Trensz, peut se localiser exclusivement dans le gros intestin et donner des dysenteries bilharziennes en général plus graves que celles produites par le Schistosoma mansoni.

Grâce au riche matériel fourni par les animaux infectés à mon laboratoire, j'ai pu réussir une expérience qui n'avait pas encore été faite, à savoir l'infection de Bullinus contortus d'élevages neufs par des œufs de schistosome provenant d'un animal infecté expérimentalement. Le 24 avril 1928, avant de partir en Corse pour effectuer une campagne antipaludique, j'ai mis au contact d'un certain nombre d'exemplaires de Bullinus le produit de broyage d'un foie de souris $\left(n^{\circ}\right.$ 517) renfermant des oufs de Schistosoma hæmatobium normaux et de nombreux œufs dégénérés. Le $1^{\text {er }}$ juin les mollusques conservés à $25^{\circ}$ et bien nourris avec de la salade n'émettaient pas encore de cercaires, mais le $1^{\text {or }}$ juillet, sur les 35 mollusques survivants, 7 étaient infectés et émettaient spontanément, dans des conditions favorables, de nombreuses cercaires avec lesquelles je fais de nouvelles expériences pour contaminer divers animaux de laboratoire. En partant des selles de hérissons renfermant toujours des œufs normaux d'où éclosent rapidement des miracidium très actifs quand on les met au contact de l'eau, je continue de nouvelles infections de mollusques, afin de pouvoir entreprendre un certain nombre d'études sur les conditions déterminant les tropismes de ces vers ainsi que sur diverses autres questions générales intéressant les médecins et les biologistes.

Le fait que les œufs de schistosomes, ayant atteint leur complet développement chez des animaux de laboratoire, sont susceptibles d'infecter les mollusques hôtes intermédiaires montre donc que dans la nature l'infection pourrait se perpétuer, en dehors de l'homme, par des animaux réceptifs qui, par leur genre de vie, pourraient entrer en contact avec les eaux souillées, soit en les buvant, soit en s'y baignant. C'est ce qui se produit certainement dans le cas du Schistosoma japonicum pour lequel l'homme n'est même qu'un hôte accidentel et peut-être aussi dans celui du Schistosoma mansoni dont la grande ubiquité à l'état adulte, dans des conditions expérimentales tout au moins, permet d'entrevoir l'existence d'hôtes animaux jouant un ròle vicariant dans la nature.

En 1917, Leiper, frappé de constater l'absence complète de Rats sauvages au bord des canaux de Marg en Egypte, où les bilharzioses 
à $S$. hæmatobium et à $S$. mansoni se contractent facilement, avait émis l'hypothèse que ces animaux devaient avoir été détruits par des infections intenses provoquées par les schistosomes. Cette hypothèse est peut-être vraie dans le cas du $S$. mansoni, qui donne des infections mortelles au rat d'après les expériences de Leiper mais elle ne peut vraisemblablement pas s'appliquer au cas de S. hæmatobium qui, dans les expériences de Cawston (1918-1919), d'Ingram (1924) et dans les miennes (1928), s'est montré un très mauvais hôte, même quand il est très jeune.

Les animaux réceptifs à la bilharziose vésicale expérimentés jusqu'à ce jour sont les suivants : singes divers (Leiper, 1917 ; Manson Bahr et Fairley, 1920 ; Archibald, 1922 ; Blacklock et Thompson, 1924 ; Brumpt, 1928), cobayes (Becker, 1916, Blacklock et Thompson, 1924), rats blancs (Leiper, 1917 ; Brumpt, 1928), souris blanches (Leiper, 1917 ; Bettencourt et Borges, 1922 ; Brumpt, 1928), hérissons (Brumpt, 1928).

Parmi ces animaux ce sont les singes asiatiques et africains, les souris et les hérissons qui sont les plus réceptifs. Les singes et les hérissons, qui semblent toujours présenter une manifestation intestinale de la bilharziose habituellement vésicale chez l'homme, sont susceptibles d'être des hôtes vicariants de cette infection. Il serait d'ailleurs intéressant d'essayer la susceptibilité de divers animaux domestiques et d'étudier la possibilité pour les œufs produits d'être évacués à l'extérieur afin de savoir quel rôle épidémiologique éven. tuel ils peuvent jouer dans la nature. J'espère pouvoir publier prochainement un travail complet dans lequel, à l'occasion des constatations anatomo-pathologiques, j'exposerai mes idées sur le mécanisme des migrations des œufs dans les tissus et les facteurs déterminant la localisation des bilharzies dans les organes de leurs hôtes expérimentaux.

\section{RÉsumé}

$1^{\circ}$ Le hérisson, dont la sensibilité aux bilharzioses ne semble pas avoir été étudiée jusqu'à ce jour, est un animal extrêmement récep. tif, tout au moins dans le cas du Schistosoma hæmatobium.

$2^{\circ}$ Cet animal, qui a présenté trois fois sur trois des lésions intestinales généralisées, ainsi que des infections hépatiques et pancréatiques intenses, n’a jamais montré de lésions vésicalles.

$3^{\circ}$ Le cycle évolutif complet, de l'œuf à l'œuf, du Schistosoma hæmatobium a été obtenu pour la première fois en partant d'une souris infectée expérimentalement. 


\section{Bibliographie}

Archibat.d (R.-G.). - Some investigations connected with the spread of Bilharziasis in the Dongola Province of the Sudan. Trans. Roy. Soc. Trop. Med. Hyg., XV, 1922-1923, p. 419-426, pl. I-V.

Becker (J.-G.). - A further Note on Bilharziosis in the Transvaal. Med. Jl. of South. A frica, XII, 1916, p. 42.

Betticount (ג.) et Borgrs (I.). - Rapport de la Mission de l'Institut Camara Pestana pour l'étude de la bilharziose au Portugal. Arq. Inst. Bact. Camara Pestana, V, 1922, p. 189230 , pl. VII-XII.

Blacklock (D.-B.) and Thompson (M.-G.). - Human schistosomiasis due to Schistosoma hamatobinm in Sierra Leone. Ann. of trop. med. and paras., XVIII, 1924, p. 211-234, pl. XIV.

Brumpt (E.) et Werblunsky (S.). - Infection expérimentale d'un mollusque de Corse (Bullinus contortus) par le Schistcsoma hamatobium, agent de la bilharziose vésicale. Bull. Acad. de med., XCIX, 1928, pp. 153-155.

Cawstox (F.-G.). - Bilharziosis in Natal. Parasitology, XI, 1918-1919, pp. 83-93.

Clapier (P.-N.). - Contribution à l'étude de la répartition des bilharzioses en Afrique équatoriale française. Bull. Soc. Path. Exot., XIII, 1920, p. 804.

DAY (H.-B.). - The etiology of egyptian splenomegaly and hepatic cirrhosis. Trans. Roy. Soc. Trop. Med. Hyg., XVIII, 1924. pl. 121.

Ingram (A.). - Note on a possible intermediate host of Schistosoma hrematobium in the Gold Coast. Ann. Trop. Med. Paras., XVIII, 1924, pp. 265-266.

LEIPER (R.-T.). - Researches on Egyptian Bilharziosis (A report to the War Office on the Results of the Bilharzia Mission in Egypt, 1915). London J. Baillière, Sons et Danielsson Ltd, 1918.

Manson-Bahr (Ph.) and FarRley (N.-H.), - Observations on bilharziasis amongst the Egyptian expeditionary forces. Parasilology, XII, 1920, p. 33-71.

Nessmann (V.) et Trensz (F.). - Nouveaux cas de bilharziose intestinale à Schislosoma hamatobium observés au Gabon. Annales de Parasitologie, V1, 1928, p. 182.

WERBLunsky (S.). - Les hôtes intermédiaires de Schistosoma hrematobium agent de la bilharziose vésicale. Thèse de la Faculté de Médécine de Paris, 1928.

Laboratoire de Parasilologie de la Faculté dé Médecine de Paris. 